\title{
Opportunistic Interference Management Increases the Capacity of Ad Hoc Networks
}

\author{
Zheng Wang ${ }^{\dagger}$, Mingyue Ji ${ }^{\dagger}$, Hamid R. Sadjadpour ${ }^{\dagger}$, J.J. Garcia-Luna-Aceves ${ }^{\ddagger}$ \\ Department of Electrical Engineering ${ }^{\dagger}$ and Computer Engineering ${ }^{\ddagger}$ \\ University of California, Santa Cruz, 1156 High Street, Santa Cruz, CA 95064, USA \\ Email:\{wzgold, davidjmy, hamid, jj\}@ soe.ucsc.edu
}

\begin{abstract}
We introduce a new multiuser diversity concept with which multiple transmitters can communicate without causing significant interference to each other. The new scheme called Opportunistic Interference Management (OIM) significantly reduces the feedback required in distributed Multiple-Input MultipleOutput (MIMO) systems, and requires an encoding and decoding complexity that is similar to that of point-to-point communications. We show that our proposed OIM scheme achieves a pernode throughput capacity of $\Theta\left(\frac{\log (T(n))}{\sqrt{n} T(n)}\right)$ in a wireless network of $n$ nodes and communication range of $T(n)=\Omega(\sqrt{\log n})$. This represents a gain of $\Theta(\log (T(n)))$ compared to simple pointto-point communication. Hence, OIM provides an alternative approach to distributed MIMO systems with significantly less feedback requirements among nodes, which makes this approach far more practical than distributed MIMO systems.
\end{abstract}

\section{INTRODUCTION}

Since the landmark work by Gupta and Kumar [1] on the capacity of wireless ad hoc networks, considerable attention has been devoted to improving or analyzing their results. Ozgur et al. [2] demonstrated that the capacity of random wireless ad hoc network scales linearly with $n$ by allowing nodes to cooperate intelligently using distributed Multiple-Input MultipleOutput (MIMO) communications. Unfortunately, distributed MIMO techniques require significant cooperation and feedback information among nodes to achieve capacity gains using multiple antenna systems. These challenges include synchronization during transmission and cooperation for decoding which makes distributed MIMO systems less practical.

As our summary of prior work in Section II indicates, like distributed MIMO solutions, all other approaches aimed at increasing the capacity of wireless networks have viewed fading and interference as major impeding factors to the scaling of wireless ad hoc networks. Prior approaches have attempted to combat interference and fading separately. Information theorists define interference into three categories. The first category is when the interference signal strength is much stronger than that of desired signal. Under this condition [3], the interference can be first decoded by the receiver and then subtracted using successive interference cancelation (SIC). Once the interference is canceled, then the desired signal can be decoded. In some applications, the interference strength is much weaker than the desired signal. Under this condition, the interference is treated as noise and the signal can be directly decoded. If the strength of interference and desired signal are comparable, a commonly used strategy is to orthogonalize the channel by means of time division multiple access (TDMA) or frequency division multiple access (FDMA) to separate the signals. Such resource allocation approach provides reliable communications for nodes in the network but decreases the capacity significantly.

Multiuser diversity [4] was introduced as an approach to increase the capacity of wireless cellular networks. The main idea behind this approach is for a base station to select a mobile station that has the best channel condition by taking advantage of the time-varying nature of fading channels, thus maximizing the signal-to-noise ratio (SNR). This idea was later extended to mobile wireless ad hoc networks [5] and MIMO cellular networks [6].

In this paper, we introduce a new multiuser diversity scheme, which we call Opportunistic Interference Management (OIM), where channel fading is used proactively to mitigate interference in the network from simultaneous transmissions, as long as there are enough nodes in the area. Just as important, OIM allows a distributed MIMO system to operate with the same level of complexity of multiple Single-Input Single-Output (SISO) systems! OIM approach is fundamentaly different from distributed cooperative MIMO systems. In distributed cooperative MIMO systems, we take advantage of MIMO systems by extending this concept to distributed systems. Clearly, such extension requires some modifications at the physical layer to overcome the challenges related to distributed space-time signal processing. OIM on the other hand, is an innovative scheduling scheme that improves concurrency in the wireless ad hoc network by allowing multiple nodes to communicate in the same channel and location and at the same time. It is therefore not surprising that this scheme requires simple point-to-point signalling for encoding and decoding. Hence, OIM is an alternative to distribued cooperative MIMO systems for increasing the throughput capacity of wireless ad hoc networks with lower complexity at the physical layer. As we will explain in more details later, this goal is achieved by proposing a new scheduling scheme for nodes in multiuser environments.

Section IV describes OIM scheme in details. Unlike all prior techniques that attempt to fight individually fading and interference as impairments in wireless channels, OIM takes advantage of one of them (fading channel) to reduce the 
negative effect of the other one (interference). By taking advantage of multiuser diversity, OIM attempts to maximize the SNR beyond a threshold, while minimizing the interferenceto-noise ratio (INR) below another threshold, such that the interference signal strength is no longer significant. The result is very effective, and constitutes a powerful technique that achieves high throughput capacity and yet requires minimum feedback and simple point-to-point encoding and decoding complexity for each node. Furthermore, we have shown [7], [8] that OIM achieves dirty paper coding capacity in wireless cellular networks. In this paper, we extend it into wireless ad hoc networks opportunistically because of no base station challenge.

Section III introduces the models used in our analysis and Section V provides the capacity analysis when OIM is used in a wireless ad hoc network. We show that the throughput capacity with OIM in wireless ad hoc networks is $C(n)=\Theta\left(\frac{\log (T(n))}{\sqrt{n} T(n)}\right)$ when $T(n)=\Omega(\sqrt{\log n})$ is the transmission range. Our approach provides a gain of $\Theta(\log (T(n))$ compared to the simple multi-hop point-topoint communications under similar network assumptions. The gain ranges from $\Theta(\log \log n)$ to $\Theta(\log n)$, depending on the value of the transmission range, while the encoding and decoding complexity of the new scheme is similar to that of point-to-point communications. The increase of the capacity is essentially because of the powerful nature of multiuser diversity in wireless fading environments.

\section{RELATED WORK}

Ozgur et al. [2] demonstrated that the capacity of random wireless ad hoc network scales linearly with $n$ by allowing nodes to cooperate intelligently using distributed MIMO communications. The distributed cooperative MIMO system is a physical layer solution for increasing concurrency in wireless ad hoc networks. One of the main drawbacks with this approach is the complexity requirement for implementing distributed cooperative MIMO systems.

Knopp and Humblet [4] derived the optimum capacity for the uplink of wireless cellular network taking advantage of multi-user diversity. They proved that if the "best" channel (i.e., the channel with the highest SNR in the network) is selected, then all of the power should be allocated to this specific user with good "channel" instead of using a water-filling power control technique. Viswanath et al in [6] used a similar idea on the downlink channel using the so called "dumb antennas" by taking advantage of opportunistic beamforming. Grossglauser et al [5] extended this multi-user diversity concept to the case of mobile ad hoc networks and took advantage of mobility of nodes to scale the capacity.

Interference is a significant impediment to the scaling of wireless networks and there are considerable efforts to mitigate its negative effects. Interference alignment [9] has been proposed to align interference to the desired signal such that the interference no longer interferes with the signal. The main idea in this approach is to use part of the degrees of freedom available at a node to transmit the information signal and the remaining part to transmit the interference. The drawback of interference alignment is that the system requires full knowledge of the channel state information (CSI). This condition is very difficult to implement in practice, and the feedback requirements of CSI are not practical for wireless ad hoc networks. Sharif and Hassibi also introduced an approach [10], [11] to search for the best SINR in a wireless cellular network. The implementation of this approach requires random beamforming and it is not clear whether this approach can be applied to wireless ad hoc networks.

In this paper, we extend the OIM scheme we have proposed previously for cellular networks [7], [8] to distributed nodes in wireless ad hoc networks. Surprisingly, by fully taking advantage of fading channels in multiuser environments, the feedback requirement is proportional to a small value, while the encoding and decoding scheme is very simple and similar to the point-to-point communications. This is achieved by utilizing an innovative scheduling scheme to allow concurrency at the physical layer. OIM does not require any changes to the physical layer while allowing multiple nodes to communicate with each other in the same channel and location and at the same time. The original multiuser diversity concept was based on looking for the best channels, while our interferencemanagement approach is based on searching simultaneously for the best and worse channels.

Xie and Kumar [12] were first to compute the information theoretic capacity of wireless ad hoc networks when the channel model is based on path loss exponent $\alpha$. They showed that for $\alpha>6$, the optimal throughput capacity of $\Theta\left(\frac{1}{\sqrt{n}}\right)$ can be achieved by nearest neighbor multihop scheme. This work was followed by others [13]-[15] to prove the optimality of the results for all values of $\alpha>4$. Finally, it was shown that hierarchical MIMO cooperation [2] can provide optimum capacity for $\alpha>3$. However, the multipath fading channel model was not considered in any of these papers and only in [2] the random phase was added to the channel model. Xue et al. [16] were first to demonstrate that multipath fading does not decrease the capacity and hence, the information theoretic upper bound throughput capacity with fading is $O\left(\frac{1}{\sqrt{n}}\right)$. Our analysis in this paper considers multipath fading for the channel model and the capacity computation is based on the generalized physical model criterion.

\section{Network Model ANd Preliminaries}

We assume a wireless network with $n$ nodes distributed randomly and uniformly in the network area. Our analysis is based on the extended network model, where the density of nodes is a constant order $\Theta(1)$ and the area of the network is a square with side length equal to $\sqrt{n}$. The capacity computation is based on extending the physical model criterion in [1] by adding fading effects in the signal-to-noise plus interference ratio (SINR) computation. To simplify our analysis, we do not consider a torus or a sphere shape for the network area. However, the results on the order capacity are the same. We assume that the node's movement causes fading. However, this 
is a restricted movement such that any node only moves within its transmission range and the network topology and routing does not change with time. If the nodes have unrestricted mobility in the entire network, we assume that the time duration that causes the topology of the network to change is always smaller than the duration for transporting information from each source to its destination. Therefore, at any snapshot during packet transmission from any source to its destination in the network, the topology is static.

Let $X_{i}$ and $X_{R(i)}$ denote the locations of node $i$ and its receiving node $R(i)$ respectively. Let $P_{i R(i)}$ be the received signal power at node $R(i)$. The wireless channel is subject to fading as described below. We define $P$ as the transmit power at node $i$ and $\left|X_{i}-X_{R(i)}\right|$ as the Euclidean distance between nodes $i$ and $R(i) . P_{i R(i)}$ is modeled as

$$
P_{i R(i)}=\left|H_{i R(i)}\right|^{2} \frac{P}{\left(\left|X_{i}-X_{R(i)}\right|\right)^{\alpha}}
$$

where $H_{i R(i)}$ is a random variable that incorporates the channel fading and $\alpha$ is the path-loss exponent whose typical values are between 2 and 6 . Under Rayleigh fading model, $H_{i R(i)}$ and $\left|H_{i R(i)}\right|^{2}$ have Rayleigh and exponential distributions respectively.

Definition 3.1: Generalized Physical Model

In this analysis, the data rate between the transmitter-receiver pair $i$ and $R(i)$ in bits/second is defined as

$$
C_{i R(i)}=W \log \left(1+\operatorname{SINR}_{i R(i)}\right),
$$

where $W$ is the bandwidth and $\operatorname{SINR}_{i R(i)}$ between the transmitting node $X_{i}$ and the receiving node $X_{R(i)}$ is defined as

$$
\operatorname{SINR}_{i R(i)}=\frac{\frac{P}{\left(\left|X_{i}-X_{R(i)}\right|\right)^{\alpha}}\left|H_{i R(i)}\right|^{2}}{N+\sum_{k \neq i} \frac{P}{\left(\left|X_{k}-X_{R(i)}\right|\right)^{\alpha}}\left|H_{k R(i)}\right|^{2}},
$$

where $N$ is the ambient noise power and $X_{k}$ 's $(k \neq i)$ are the interfering nodes. Note that the channel model consists of large scale fluctuation $\left|X_{i}-X_{R(i)}\right|^{-\alpha}$ and small scale fluctuation $\left|H_{k R(i)}\right|^{2}$.

The throughput of $C(n)$ bits per second for each node is feasible if there is a spatial and temporal scheme for scheduling transmissions, such that, by operating the network in a multi-hop fashion and buffering at intermediate nodes when awaiting transmission, every node can send $C(n)$ bits per second on average to its destination node. $C(n)$ is said to be of order $\Theta(f(n))$ bits/second if there exists $c_{2}>c_{1}>0$ such that $c_{1} f(n)<C(n)<c_{2} f(n)$.

To simplify the analysis, we assume the node density is equal to unity. Hence, if $|S|$ denotes the area of space region $S$, the expected number of the nodes, $E\left(N_{S}\right)$, in this area is given by $E\left(N_{S}\right)=|S|$. Let $N_{j}$ be a random variable defining the number of nodes in $S_{j}$. Then, for the family of variables $N_{j}$, we have the following standard results known as the Chernoff bound [17].

$$
P\left[\left|N_{j}-\right| S_{j}||>\delta\left|S_{j}\right|\right]<e^{-\theta\left|S_{j}\right|},
$$

where $\theta$ is some constant value depending $\delta$ and $\delta$ is a positive arbitrarily small value close to zero.

All nodes in the network are endowed with a single antenna. In some area, there are $K$ transmitters which want to transmit information while the rest of the nodes around them are the potential receivers. If the total number of transmitters and receivers is $m$, then the channel matrix $\mathbf{H}$ is a $(m-K) \times K$ matrix with elements $h_{j i}$ for $j \in[1,2, \cdots, m-K]$ and $i \in[1,2, \cdots, K]$ are the receiver and transmitter index respectively. We consider block fading model where the channel coefficients are constant during coherence interval of $\mathbf{T}$. Then the received signal $\mathbf{Y}^{(m-K) \times 1}$ can be expressed as

$$
\mathbf{Y}=\mathbf{H} \mathbf{x}+\mathbf{n}
$$

where $\mathbf{x}$ is $K \times 1$ transmit signal vector and $\mathbf{n}$ is $(m-K) \times 1$ noise vector. The noise at each of the receivers is i.i.d. with $\mathcal{C N}\left(0, \sigma_{n}^{2}\right)$ distribution.

\section{Opportunistic InTERFEREnCE MANAGEMENT (OIM)}

\section{A. Scheduling Protocol}

Fig. 1 illustrates the system involved in OIM. Without loss of generality, we assume that the receiver $R(i)=i$ for $i \in$ $[1,2, \ldots, K]$ in Fig. 1 . In this figure, solid line and dotted line represent a strong and weak channel between transmitters and receivers respectively.

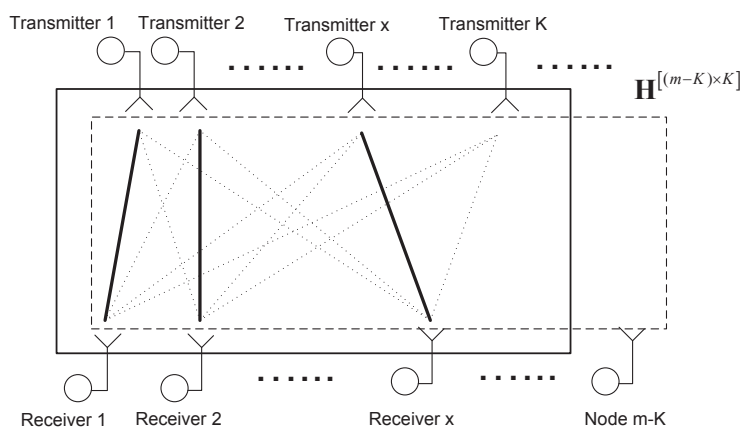

Fig. 1. Opportunistic Interference Management System Model

We assume that, for each node, there is always some traffic demand to any other neighbor node at any time slot. Each packet is either destined for a particular neighbor node or is relayed through a route that need the node to relay.

For any time slot $\mathbf{T}$, there are $x$ potential transmitters out of $K$ that satisfy the OIM condition, where $x$ is a random variable with mean value of $D=\mathbf{E}(x)$. We will define subsequently the probability distribution function of $x$ and the relationship between $D, K$ and the rest of the nodes $m-K$. In practice, we select $K$ transmitter nodes who are close by in order to make coordination easier. During the first phase of communication, the $K$ transmitters sequentially transmit $K$ pilot signals. In this period, all the other $m-K$ nodes listen to these known messages. After the last pilot signal is transmitted, all of the other nodes evaluate the SNR for each transmitter. If the 
SNR for only one transmitter is greater than a pre-determined threshold $\mathrm{SNR}_{\text {tr }}$ and below another pre-determined threshold of $\mathrm{INR}_{\mathrm{tr}}$ for the remaining $K-1$ transmitters, that particular receiver selects that particular transmitter. In the second phase of communication, these receivers notify the transmitters that they have the required criterion to receive packets during the remaining time period of $\mathbf{T}$. If appropriate values for $\mathrm{SNR}_{\mathrm{tr}}$ and $\mathrm{INR}_{\mathrm{tr}}$ are chosen, such that $\mathrm{SNR}_{\mathrm{tr}} \gg \mathrm{INR}_{\mathrm{tr}}$, then the transmitters can transmit different packets to different receivers concurrently. The receivers only receive their perspective packets with strong signal and can treat the rest of packets as noise. The value of $\mathrm{SNR}_{\mathrm{tr}}$ (or $\mathrm{INR}_{\mathrm{tr}}$ ) can be selected as high (or low) as required for a given system as long as $m$ is large enough. We will show their relationship in details later.

In the following analysis, our objective is that for any given number of nodes $m$, fading parameter $\sigma$, and SINR $_{\text {tr }}$ requirement, we compute the value of $D=\mathbf{E}(x)$.

\section{B. Theoretical Analysis}

Let's define $\mathrm{SNR}_{i R(i)}$ and $\mathrm{INR}_{j R(i)}$ as the signal-to-noise ratio and interference-to-noise ratio between transmitter $i$, other transmitter $j, j \neq i$ and $i$ 's corresponding receiver $R(i)$ respectively. Note that we only consider fading (small scale fluctuation of channel) for the analysis of OIM as explained earlier. The objective of OIM is to find $x$ receiver nodes out of $m-K$ choices to satisfy the following criteria. Since $x$ is a random variable, we use the average value of $x$ receiver that satisfies OIM requirement, i.e., $D=\mathbf{E}(x)$. Then for any associate transmitter $i, i \in 1,2, \cdots, K, i$ 's corresponding receiver $R(i)$ and other transmitter $j, j \in 1,2, \cdots K, j \neq i$, there has

$$
\begin{aligned}
& \mathrm{SNR}_{i R(i)} \geq \mathrm{SNR}_{\mathrm{tr}}, i \in, 1,2, \cdots, K, R(i) \in 1,2, \cdots, x \\
& \mathrm{INR}_{j R(i)} \leq \mathrm{INR}_{\mathrm{tr}}, j \in 1,2, \cdots, K, j \neq i
\end{aligned}
$$

The above condition states that each one of the $x$ receiver nodes has a very good channel to a single transmitter node and weak channel (strong fading) to the other $K-1$ receiver nodes as shown in Fig. 1.

Then, we define $\operatorname{SINR}_{i R(i)}$ as

$$
\operatorname{SINR}_{i R(i)}=\frac{\operatorname{SNR}_{i R(i)}}{\sum_{j=1, j \neq i}^{K-1} \operatorname{INR}_{j R(i)}+1},
$$

and SINR $_{\text {tr }}$ as

$$
\operatorname{SINR}_{\mathrm{tr}}=\frac{\mathrm{SNR}_{\mathrm{tr}}}{(K-1) \mathrm{INR}_{\mathrm{tr}}+1}
$$

respectively.

Hence, supposing we can find $D=\mathbf{E}(x)$ transmitterreceiver pairs satisfying Eq. (6), then the sum rate can be written as

$$
\begin{aligned}
C_{\text {proposed }} & =\sum_{i=1}^{D} \log \left(1+\operatorname{SINR}_{i R(i)}\right), \\
& =\sum_{i=1}^{D} \log \left(1+\frac{\operatorname{SNR}_{i R(i)}}{\sum_{j=1, j \neq i}^{K-1} \mathrm{INR}_{j R(i)}+1}\right), \\
& \geq D \log \left(1+\frac{\mathrm{SNR}_{\mathrm{tr}}}{(K-1) \mathrm{INR}_{\mathrm{tr}}+1}\right), \\
& =D \log \left(1+\mathrm{SINR}_{\mathrm{tr}}\right)
\end{aligned}
$$

In the following, we will prove that for any given value of $\mathrm{SINR}_{\mathrm{tr}}$, there exists a relationship between $m$ and $D$ that will satisfy Eq. (9). To prove the existence of this algorithm, we need to prove that there are $D=\mathbf{E}(x)$ transmitter-receiver pairs that satisfy Eq. (6) on average.

To prove the condition in Eq. (9), we assume that the channel distribution is Rayleigh fading channel. However, any time-varying channel model can be utilized for the following derivations. Note that for a Rayleigh fading channel $\mathbf{H}$ distribution, the probability distribution of SNR is given by [18]

$$
p(\mathrm{z})= \begin{cases}\frac{1}{\sigma} \exp \left(-\frac{z}{\sigma}\right), & z>0 \\ 0, & z \leq 0\end{cases}
$$

where $z$ is the SNR (or INR) value and $E_{\mathbf{H}}(z)=\sigma$, $\operatorname{Var}_{\mathbf{H}}(z)=\sigma^{2}$. Equivalently, $\sqrt{\sigma / 2}$ is the parameter for Rayleigh fading distribution which shows the strength of the fading channel.

Assuming the probability distribution function, expected value and variance of $x$ are $\operatorname{Pr}(x), D=\mathbf{E}(x)$ and $\Delta^{2}=$ $\operatorname{Var}(x)$ respectively. Note that by selecting the average value of $x$, in practice the actual number of nodes satisfying OIM is either larger or smaller than this average value. Therefore, we may decide to choose a constant value such that with a probability arbitrarily close to zero, the actual number of nodes satisfying OIM criterion is always smaller than this value. By utilizing Chebyshev's inequality, we have

$$
\operatorname{Pr}\left(|x-D| \geq c_{0} \Delta\right) \leq \frac{1}{c_{0}^{2}} .
$$

This equation implies that for any given $c_{0}$, the value of $x$ is smaller than $D+c_{0} \Delta$ with probability greater than $1-\frac{1}{c_{0}^{2}}$. Clearly this probability can be selected arbitrarily close to one. The practical price is increase in transmission of pilot signals during the first phase of communications. In the followings, we will prove that $D=\Theta(K)$.

\section{Capacity Analysis of Ad Hoc Networks with OPPORTUNISTIC INTERFERENCE MANAGEMENT}

Our achievable bound for the capacity analysis is based on the TDMA scheme that was originally introduced in [19]. In this approach, the network is divided into smaller square cells each one with an area such that all the nodes inside each cell are connected. Therefore, each square cell has an area of $T^{2}(n) / 2$ which makes the diagonal length of square equal to $T(n)$ as shown in Fig. 2. Under this condition, if 


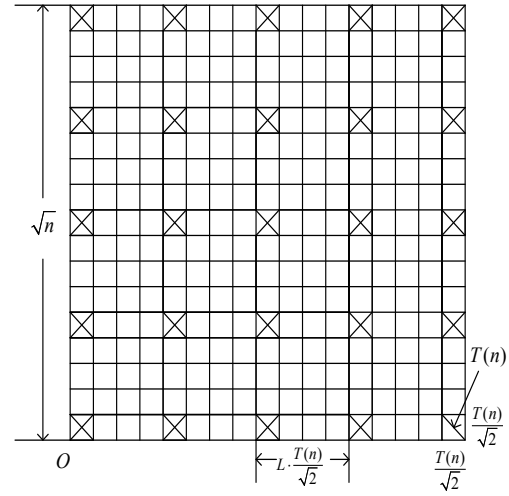

Fig. 2. The cell construction in extended wireless ad hoc network

the transmission range is at most $T(n)$ for each hop, then all nodes inside a cell are within cooperation range of each other. We build a cell graph over the network that are occupied with at least one vertex (node) [19]. We organize cells into groups such that simultaneous transmissions within each group does not violate the OIM condition for successful communication. Let $L$ represent the minimum number of cell separations in each group of cells that communicate simultaneously. In every $1 / L^{2}$ time slots, each cell receives one time slot to communicate. In an active cell, each transmitter node either sends a packet to one of the nodes inside the cell or a node in adjacent cells. Fig. 2 shows a group of active cells with cross symbol inside the cells. Note that the distance between interfering cells is at least $q T(n) L / \sqrt{2}-T(n) / \sqrt{2}$ for $q=1,2, \cdots$, centered around any active cell.

Our analysis is based on computing SINR for two cases of interference within a cell and interference from outside the cell. We denote the former one as $\mathrm{SINR}_{\text {inner }}$ and the latter as $\mathrm{SINR}_{\text {outer }}$. Note that in general, the SNR can be computed as

$$
\mathrm{SNR}_{i R(i)}=\frac{\frac{P}{\left|X_{i}-X_{R(i)}\right|^{\alpha}}\left|H_{i R(i)}\right|^{2}}{N} \geq \mathrm{SNR}_{\mathrm{tr}} .
$$

The lower bound is derived based on the OIM condition. If we assume $\left|H_{i R(i)}\right|^{2} \geq c_{3}$, then $c_{3}$ can be selected as $c_{3}=\mathrm{SNR}_{\operatorname{tr}} N \frac{\left|X_{i}-X_{R(i)}\right|^{\alpha}}{P}$. To compute the lower bound for SINR $_{\text {outer }}$, we note that $\mathbf{E}\left[\left|H_{k R(i)}\right|^{2}\right]=\sigma$ and $\operatorname{Var}\left[\left|H_{k R(i)}\right|^{2}\right]=\sigma^{2}$ because of the characteristic of exponential distribution $\left|H_{k R(i)}\right|^{2}$ for any $k$ in Eq. (10). Due to $\left|X_{i}-X_{R(i)}\right| \leq T(n)$ for neighbor cell, then

$$
\begin{aligned}
\operatorname{SINR}_{\text {outer }} & =\frac{\frac{P}{X_{i}-\left.X_{R(i)}\right|^{\alpha}}\left|H_{i R(i)}\right|^{2}}{N+\sum_{k \neq i} \frac{P}{\left|X_{k}-X_{R(i)}\right|^{\alpha}}\left|H_{k R(i)}\right|^{2}} \\
& \geq \frac{\frac{c_{3} P}{(T(n))^{\alpha}}}{N+\sum_{q=1}^{\infty} 8 q \frac{P}{(q T(n) L / \sqrt{2}-T(n) / \sqrt{2})^{\alpha}}\left|H_{q R(i)}\right|^{2}} \\
& =\frac{\frac{c_{3} P}{(\sqrt{2})^{\alpha}}}{N\left(\frac{T(n)}{\sqrt{2}}\right)^{\alpha}+\frac{8 P}{L^{\alpha}} \sum_{q=1}^{\infty} \frac{q}{\left(q-\frac{1}{L}\right)^{\alpha}}\left|H_{q R(i)}\right|^{2}}(13)
\end{aligned}
$$

where, we need to prove the second term of denominator bounded provided that $P$ increases with $T(n)$ in extended networks. We define $S_{q}=\frac{q}{\left(q-\frac{1}{L}\right)^{\alpha}}$. The sum $S=\sum_{q=1}^{\infty} S_{q}$ is bounded by a constant $c_{4}$ as follows [20] when $\alpha>2$.

$$
\begin{aligned}
S= & \sum_{q=1}^{\infty} \frac{1}{\left(q-\frac{1}{L}\right)^{\alpha-1}}+\frac{1}{L} \sum_{q=1}^{\infty} \frac{1}{\left(q-\frac{1}{L}\right)^{\alpha}} \\
\leq & \frac{1}{\left(1-\frac{1}{L}\right)^{\alpha-1}}+\int_{1-\frac{1}{L}}^{\infty} \frac{1}{x^{\alpha-1}} d x \\
& +\frac{1}{L}\left(\frac{1}{\left(1-\frac{1}{L}\right)^{\alpha}}+\int_{1-\frac{1}{L}}^{\infty} \frac{1}{x^{\alpha}} d x\right)=c_{4}
\end{aligned}
$$

When $L$ is selected sufficiently large, then the effect of interference from outside cells can be reduced to any desired value based on Eq. (13). Next we need to prove that $S=\sum_{q=1}^{\infty} S_{q}\left|H_{q R(i)}\right|^{2}$ is bounded. Because $\left|H_{q R(i)}\right|^{2}$ is a random variable, then we should prove $\operatorname{Pr}\left(\sum_{q=1}^{\infty} S_{q}\left|H_{q R(i)}\right|^{2} \rightarrow \infty\right)=0$.

Let's define $\mathbf{E}\left[\left|H_{q R(i)}\right|^{2}\right]=\sigma$, then

$\mu=\mathbf{E}\left[\sum_{q=1}^{\infty} S_{q}\left|H_{q R(i)}\right|^{2}\right]=\sum_{q=1}^{\infty} S_{q} \mathbf{E}\left[\left|H_{q R(i)}\right|^{2}\right] \leq c_{4} \sigma$.

From Chebyshev's inequality,

$$
\operatorname{Pr}\left(\left.\left|\sum_{q=1}^{\infty} S_{q}\right| H_{q R(i)}\right|^{2}-\mu \mid \geq \alpha\right) \leq \frac{V^{2}}{\alpha^{2}}
$$

where, $V=\operatorname{Var}\left[\sum_{q=1}^{\infty} S_{q}\left|H_{q R(i)}\right|^{2}\right]$. Because of $S_{q}^{2} \leq$ $S_{q}, \forall q$, then $V=\sum_{q=1}^{\infty} S_{q}^{2} \operatorname{Var}\left[\left|H_{q R(i)}\right|^{2}\right] \leq c_{4} \sigma^{2}$ provided that $\left|H_{q R(i)}\right|$ are i.i.d for different values of $q$ in extended network model. Clearly, if $\alpha \rightarrow \infty$, then

$$
\operatorname{Pr}\left(\sum_{q=1}^{\infty} S_{q}\left|H_{k R(i)}\right|^{2} \rightarrow \infty\right)=0
$$

Thus, the Eq. (13) is bounded as

$$
\mathrm{SINR}_{\text {outer }} \geq \mathrm{SINR}_{\text {tr }}(\text { outer })
$$

where, $\operatorname{SINR}_{\mathrm{tr}}$ (outer) is a constant term derived from Eq. (13) that is defined based on the communication requirements for each node. From Eq. (8), the lower bound for SINR $_{\text {inner }}$ is given by

$$
\mathrm{SINR}_{\mathrm{inner}} \geq \mathrm{SINR}_{\mathrm{tr}}=\operatorname{SINR}_{\mathrm{tr}}(\text { inner }) .
$$

Combining Eq. (18) and Eq. (19), SINR is given by

$$
\begin{aligned}
& \operatorname{SINR}_{i R(i)}=\frac{\text { Signal }}{N+\text { Interference }_{\text {outer }}+\text { Interference }_{\text {inner }}}, \\
& \geq \frac{\text { Signal }}{N+\frac{\text { Signal }}{\operatorname{SINR}_{\text {tr }}(\text { outer })}-N+\frac{\text { Signal }}{\operatorname{SINR}_{\text {tr }}(\text { inner })}-N}, \\
& \geq \frac{1}{\frac{1}{\operatorname{SINR}_{\text {tr }} \text { (outer) }}+\frac{1}{\operatorname{SINR}_{\text {tr }} \text { (inner) }}}=\operatorname{SINR}_{\text {tr }}(\operatorname{tota}(20)
\end{aligned}
$$

Next, we derive the relationship between $D=\mathbf{E}(x), K$ and $m=\Theta\left(T^{2}(n)\right)$ in order to compute the throughput capacity 
for each cell. Based on Eq. (9), the order capacity for each cell can be computed.

Let's define event $A$ is for a receiver node that satisfies the condition in Eq. (6), and assume that the channels between the transmitter and receiver nodes are i.i.d. in extended network model, then this probability can be derived as

$$
\begin{aligned}
\operatorname{Pr}(A) & =\left(\begin{array}{c}
K \\
1
\end{array}\right) \int_{\mathrm{SNR}_{\mathrm{tr}}}^{\infty} p(z) d z\left(\int_{0}^{\mathrm{INR}} p(z) d z\right)^{K-1} . \\
& =K e^{-\frac{\mathrm{SNR}_{\mathrm{tr}}}{\sigma}}\left(1-e^{-\frac{\mathrm{INR}_{\mathrm{tr}}}{\sigma}}\right)^{K-1} .
\end{aligned}
$$

Note that $\operatorname{Pr}(A)$ is the probability of a receiver node satisfying condition in Eq. (6) for any one of the transmitter nodes. Our objective is to maximize this probability based on network parameters. Maximizing $\operatorname{Pr}(A)$ will maximize the number of OIM nodes which is a function of the total number of nodes $m$. Note that among all network parameters $m, \mathrm{SNR}_{\mathrm{tr}}, \mathrm{INR}_{\mathrm{tr}}$, and $\sigma$, the values of $m$ and $\sigma$ are really related to the physical properties of the network and are not design parameters. Further, the parameters $\mathrm{SNR}_{\mathrm{tr}}$ and $\mathrm{INR}_{\mathrm{tr}}$ can be replaced with a single parameter SINR $_{\text {tr }}$ using Eq. (8).

Remember $x$ is a random variable that denotes the number of receiver nodes satisfying the OIM condition, i.e., each receiver node has a very strong channel with any one transmitter node and very weak channel (deep fade) with all other $K-1$ transmitter nodes. Note that it is possible that two receiver nodes satisfy OIM condition for the same transmitter. Thus, we define $y$ is the random variable satisfying satisfying Eq. (6).

The event that $y=d$ receiver nodes satisfy the OIM constraint satisfies binomial distribution as follows:

$$
\operatorname{Pr}(y=d)=\left(\begin{array}{c}
m-K \\
d
\end{array}\right)(\operatorname{Pr}(A))^{d}(1-\operatorname{Pr}(A))^{m-K-d} .
$$

Note that there is some probability that for the difference receivers, they are associated with the same transmitter. We will use the following approach to compute the lower bound of the capacity we achieved. We will see it does not affect the order of the capacity. The probability that the first receiver associated to any of the antennas at the base station is $\operatorname{Pr}(A)$, and this probability for the second receiver is $\frac{K-1}{K} \operatorname{Pr}(A)$. This probability can be similarly computed for all other receivers. The probability for the last receiver $d^{\text {th }}$ to satisfy Eq. (6) is $\frac{K-d+1}{K} \operatorname{Pr}(A)>\frac{1}{K} \operatorname{Pr}(A)$. From this argument, it is clear that these probabilities are lower bounded as $\frac{1}{K} \operatorname{Pr}(A)$.

The lower bound for the expected value of $x$ is given by

$$
D=\mathbf{E}(x) \geq \frac{m-K}{K} \operatorname{Pr}(A) .
$$

It is noteworthy to mention again that the number of receivers that satisfy OIM condition $x$ is a random variable and $D$ is simply the average value of this random variable. Thus,

$$
m \leq K\left(D(\operatorname{Pr}(A))^{-1}+1\right) .
$$

Note that $m$ is upper bounded by the inverse of $\operatorname{Pr}(A)$. Therefore, in order to minimize $m$, it is necessary to minimize
$(\operatorname{Pr}(A))^{-1}$ given $\mathrm{SINR}_{t r}$ condition in Eq. (8).

$$
\begin{array}{ll}
\text { minimize } & (\operatorname{Pr}(A))^{-1} \\
\text { subject to } & \mathrm{SINR}_{\mathrm{tr}}=\frac{\mathrm{SNR}_{\mathrm{tr}}}{(K-1) \mathrm{INR}_{\mathrm{tr}}+1}
\end{array}
$$

This optimization problem can be rewritten as

$$
\begin{aligned}
& \min _{E q \cdot(26)}\left((\operatorname{Pr}(A))^{-1}\right) \\
& =\frac{1}{K} \min _{E q \cdot(26)}\left(\frac{e^{\frac{\mathrm{SNR}_{\mathrm{tr}}}{\sigma}}}{\left(1-e^{-\frac{\mathrm{INR_{ \mathbb {H } }}}{\sigma}}\right)^{K-1}}\right) \text {, }
\end{aligned}
$$

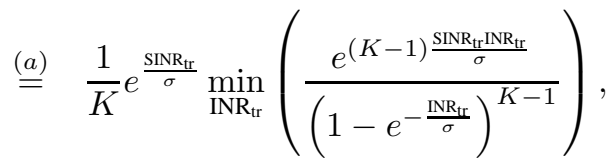

$$
\begin{aligned}
& \stackrel{(b)}{=} \frac{1}{K} e^{\frac{\mathrm{SINR}_{\mathrm{tr}}}{\sigma}} \sigma^{K-1} \min _{\mathrm{INR}_{\mathrm{tr}}}\left(\frac{e^{(K-1) \frac{\mathrm{SINR}_{\mathrm{t}} \mathrm{INR}_{\mathrm{tr}}}{\sigma}}}{\left(\mathrm{INR}_{\mathrm{tr}}\right)^{K-1}}\right) \text {. }
\end{aligned}
$$

We derive the equality (a) by replacing $\mathrm{SNR}_{\mathrm{tr}}$ with $\mathrm{INR}_{\mathrm{tr}}$ and $\mathrm{SINR}_{\text {tr }}$ using Eq. (8). Since in practice a successful communication occurs when we have a predetermined minimum value for SINR, therefore we fix the value of $S_{N N R}$ tr and attempt to optimize the above equation based on $\mathrm{INR}_{\mathrm{tr}}$. The limitation in (b) is derived by assuming $\frac{\mathbb{I N R}_{t}}{\sigma} \rightarrow 0$ and the fact that $\lim _{x \rightarrow 0}(1-\exp (-x))=x$. Note that the unique characteristic of this new scheme is to take advantage of strong fading and clearly, under that circumstance the value of $\frac{I^{N} R_{\text {tr }}}{\sigma}$ is small.

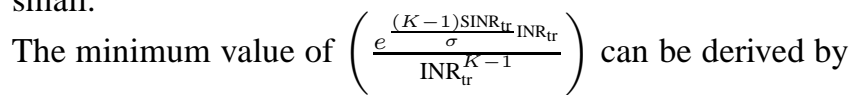
taking its first derivative with respect to $\mathrm{INR}_{t r}$ and making it equal to zero.

$$
\begin{aligned}
& e^{\frac{(K-1) \operatorname{SINR}_{\mathrm{tr}}}{\sigma} \mathrm{INR}_{\mathrm{tr}}} \times \\
& \left(\frac{(K-1) \operatorname{SINR}_{\mathrm{tr}}}{\sigma} \mathrm{INR}_{\mathrm{tr}}^{K-1}-(K-1) \mathrm{INR}_{\mathrm{tr}}^{K-2}\right)=0
\end{aligned}
$$

The solution for $\mathrm{INR}_{t r}^{*}$ is

$$
\mathrm{INR}_{\mathrm{tr}}^{*}=\frac{\sigma}{\operatorname{SINR}_{\mathrm{tr}}} .
$$

Then with optimum value for $(\operatorname{Pr}(A))^{-1}$ using Eq. (27), the optimum $m$ is derived from Eq. (24) as

$$
m \leq K+D e^{\frac{\text { IINR tr }_{\sigma}}{\sigma}}\left(\operatorname{SINR}_{\mathrm{tr}} e\right)^{K-1} .
$$

This value is derived by replacing the optimum value of $\mathrm{INR}_{\mathrm{tr}}^{*}$ into Eq. (27) and using the limitation (b) in this equation.

Now we investigate the asymptotic behavior of the network (i.e. $m \rightarrow \infty$ ) and try to compute the maximum achievable capacity and scaling laws for this scheme. Note that $\sigma$ represents the strength of fading channel and as this parameter increases or equivalently the channel experience more severe fade, then the value of $D$ increases. The main reason is the fact that fading environment helps to combat interference.

Now we investigate the asymptotic behavior of the network (i.e. $n \rightarrow \infty$ ) and try to compute the maximum achievable 
capacity and scaling laws for this scheme. From Eq. (30), if we select $D=\Theta(K)$, then we have

$$
m=O\left(D e^{K}\right)=O\left(K e^{K}\right)=O\left(e^{2 K}\right)
$$

Thus, when $m=\Theta\left(T^{2}(n)\right)$,

$$
D=\Theta(K)=\Theta(\log m)=\Theta(\log T(n))
$$

Then by utilizing Eq. (9), the scaling laws of OIM scheme for each cell is

$$
\begin{aligned}
\mathbf{E}\left(C_{\text {proposed }}\right) & =\Theta\left(D \log \left(1+\operatorname{SINR}_{\text {tr }}\right)\right) \\
& =\Theta(\log T(n))
\end{aligned}
$$

It is worthy to point out that when $\sigma$ tends to zero, this technique cannot achieve the optimum value of $K$. Equivalently, this condition occur when the channel fading is not strong. This is contrary to the current belief for point-to-point communications that fading reduces the network capacity. In a multi-user environment, fading actually is very helpful. Our proposed multi-user diversity scheme also is different from the original scheme that requires the transmitter to search for the node with the best channel condition. As we have shown, fading is very important and when the channel fading strength increases, we can achieve better capacity performance in the network.

Next we prove that when $n$ nodes are distributed uniformly over a square area, each cell contains $\Theta\left(T^{2}(n)\right)$ nodes w.h.p. The objective is to find an achievable bound using the Chernoff bound, such that the distribution of the number of nodes in each cell space is sharply concentrated around its mean.

Lemma 5.1: The square cells of side length $T(n) / \sqrt{2}$ for concurrent transmission contains $\Theta\left(T^{2}(n)\right)$ nodes w.h.p., and is uniformly distributed for all $j$ cells, $1 \leq j \leq\left[\frac{n}{(L T(n) / \sqrt{2})^{2}}\right\rceil$, when $T(n)=\Omega(\sqrt{\log n})$.

Proof: The statement of this lemma can be expressed as

$$
\lim _{n \rightarrow \infty} P\left[\bigcap_{j=1}^{\left[\frac{n}{(L T(n) / \sqrt{2})^{2}}\right\rceil}\left|N_{j}-E\left(N_{j}\right)\right|<\delta E\left(N_{j}\right)\right]=1,
$$

where $N_{j}$ and $E\left(N_{j}\right)$ are the random variables that represent the number of nodes in the square cell with diagonal distance of $T(n)$ centered around cell $j$ and the expected value of this random variable respectively, and $\delta$ is a positive arbitrarily small value close to zero.

From the Chernoff bound in Eq. (4), for any given $0<\delta<1$, we can find $\theta>0$ depending $\delta$ such that $P\left[\left|N_{j}-E\left(N_{j}\right)\right|>\delta E\left(N_{j}\right)\right]<e^{-\theta E\left(N_{j}\right)}$. Thus, we can conclude that the probability that the value of the random variable $N_{j}$ deviates by an arbitrarily small constant value from the mean tends to zero as $n \rightarrow \infty$. This is a key step in showing that when all the events $\bigcap_{j=1}^{\left\lceil\frac{n}{(L T(n) / \sqrt{2})^{2}}\right\rceil}\left|N_{j}-E\left(N_{j}\right)\right|<$ $\delta E\left(N_{j}\right)$ occur simultaneously, then all $N_{j}$ 's converge uniformly to their expected values. Utilizing the union bound, we arrive at

$$
\begin{aligned}
& P\left[\bigcap_{j=1}^{\left[\frac{n}{(L T(n) / \sqrt{2})^{2}}\right\rceil}\left|N_{j}-E\left(N_{j}\right)\right|<\delta E\left(N_{j}\right)\right] \\
& \geq 1-\sum_{j=1}^{\left\lceil\frac{n}{(L T(n) / \sqrt{2})^{2}}\right\rceil} P\left[\left|N_{j}-E\left(N_{j}\right)\right|>\delta E\left(N_{j}\right)\right] \\
& >1-\left\lceil\frac{n}{(L T(n) / \sqrt{2})^{2}}\right\rceil e^{-\theta E\left(N_{j}\right)} \text {. }
\end{aligned}
$$

Given that $E\left(N_{j}\right)=\frac{T^{2}(n)}{2}$, then we have

$$
\begin{aligned}
& \lim _{n \rightarrow \infty} P\left[\bigcap_{j=1}^{\left\lceil\frac{n}{(L T(n) / \sqrt{2})^{2}}\right\rceil}\left|N_{j}-E\left(N_{j}\right)\right|<\delta E\left(N_{j}\right)\right] \\
& \geq 1-\lim _{n \rightarrow \infty}\left\lceil\frac{n}{(L T(n) / \sqrt{2})^{2}}\right\rceil e^{-\theta T^{2}(n) / 2}
\end{aligned}
$$

If $T(n) \geq \sqrt{2 \log n / \theta}, \lim _{n \rightarrow \infty} \frac{n e^{-\theta T^{2}(n) / 2}}{T^{2}(n)} \rightarrow 0$, which completes the proof.

Next we discuss the routing scheme to achieve the achievable lower bound capacity which is similar with the routing scheme in [20]. We extend this routing scheme from the dense-network model into the extended-network model to accommodate fading. According to the model, each node $i, 1 \leq i \leq n$, generates data packets at a rate $C(n)$ with each destination chosen as the node nearest to a randomly chosen location $Y_{i}$. Denote by $X_{\text {dest }(i)}$ the node nearest to $Y_{i}$, and by $L_{i}$ the straight-line segment connecting $X_{i}$ and $Y_{i}$ (see Fig. 3). The packets generated by $X_{i}$ are forwarded toward $X_{\text {dest(i) }}$ in a multi-hop fashion, from cell to cell in the order that they are intersected by $L_{i}$. In each hop, the packet is transmitted from one cell to the next cell intersecting $L_{i}$. Any node in the cell can be chosen as a receiver. Finally, after reaching the cell containing $Y_{i}$, the packet will be forwarded to $X_{\text {dest }(i)}$ in the next active slot for that cell. This can be done because $X_{\text {dest (i) }}$ is within a range of $T(n)$ to any node in that cell. There is a bound on the number of routes each cell needs to serve, which means we bound the probability that a line will intersect a particular cell.

For completeness, we present the following two lemmas for the extended network.

Lemma 5.2: For every line $L_{i}$ and cell $S_{k_{0}, j_{0}}$,

$$
\operatorname{Pr}\left\{\text { Line } L_{i} \text { intersects } S_{k_{0}, j_{0}}\right\}=p=O\left(\frac{T(n)}{\sqrt{n}}\right)
$$

Proof: We define $S_{k_{0}, j_{0}}$ as the cell which is contained in a disk of radius $T(n) / 2$ centered at $D$ as shown in Fig. 3 . Suppose $X_{i}$ is at distance $x$ from the disk. We extend the two tangent lines originating from $X_{i}$ equally such that $\left|X_{i} A\right|=$ $\left|X_{i} B\right|$ and $\left|X_{i} C\right|=\sqrt{2 n}$, where $C$ is the mid-point of $A B$.

Then $L_{i}$ intersects $S_{k_{0}, j_{0}}$ only if $Y_{i}$ is in the shaded area. Its area is less than the minimum of $n$ and the area of the triangle, which is $\sqrt{2 n} \times \frac{\sqrt{2 n} \frac{T(n)}{2}}{\sqrt{(x+T(n) / 2)^{2}-(T(n) / 2)^{2}}}<n T(n) / x$. 


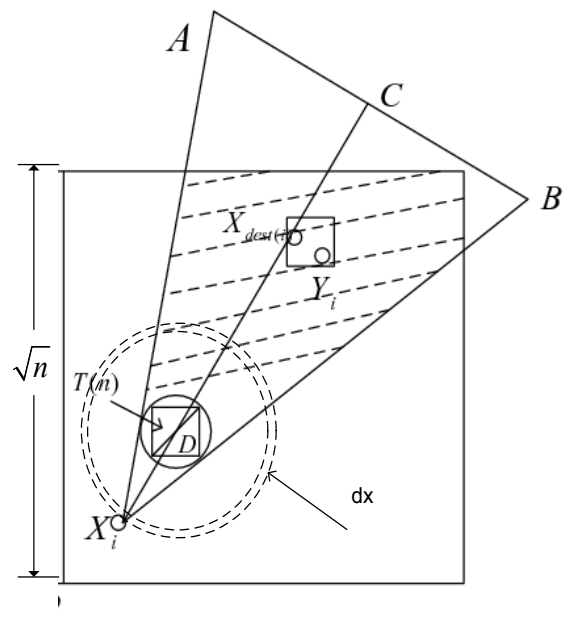

Fig. 3. Routing scheme proof

The location of $X_{i}$ is uniformly distributed, therefore, the probability density function that $X_{i}$ is at distance $x$ from the disk is a ring that is bounded by $O\left(\frac{x+T(n) / 2}{n} d x\right)$. Hence,

$\operatorname{Pr}\left\{\right.$ Line $L_{i}$ intersects $\left.S_{k_{0}, j_{0}}\right\}$,

$$
\begin{aligned}
& =O\left(\frac{1}{n} \int_{T(n) / 2}^{\sqrt{2 n}}(\min (n T(n) / x, n))\left(\frac{x+T(n) / 2}{n}\right) d x\right) \\
& =O\left(\frac{T(n)}{\sqrt{n}}\right) .
\end{aligned}
$$

Based on the above lemma, we can show the following uniform bound on the number of routes served by each cell.

Lemma 5.3: It can be proved that

$$
\begin{aligned}
& \lim _{n \rightarrow \infty} \operatorname{Pr}\left(\sup _{(k, j)}\left\{\text { Number of lines } L_{i} \text { intersecting } S_{k, j}\right\}\right. \\
& =O(\sqrt{n} T(n)))=1
\end{aligned}
$$

Proof: First we derive the bound for the number of routes served by one particular cell $S_{k_{0}, j_{0}}$. Define i.i.d. random variable $I_{i}, i \leq i \leq n$, as follows.

$$
I_{i}=\left\{\begin{array}{l}
1, \text { if } L_{i} \text { intersects } S_{k_{0}, j_{0}} \\
0, \text { if not }
\end{array}\right.
$$

Let $\operatorname{Pr}\left(I_{i}=1\right)=p \forall i$, where $p$ is defined in Lemma 5.2. Denote $Z_{n}$ the total number of routes served by $S_{k_{0}, j_{0}}$. Then $Z_{n}:=I_{1}+I_{2}+\cdots+I_{n}$. Using Chernoff bound, for all positive values of $b$ and $a, \operatorname{Pr}\left(Z_{n}>b\right) \leq \frac{E\left[e^{a Z_{n}}\right]}{e^{a b}}$. Since $1+x \leq e^{x}$, we have

$$
\begin{aligned}
E\left[e^{a Z_{n}}\right] & =\left(1+\left(e^{a}-1\right) p\right)^{n} \leq \exp \left(n\left(e^{a}-1\right) p\right), \\
& =O\left(\exp \left(\left(e^{a}-1\right) \sqrt{n} T(n)\right)\right) .
\end{aligned}
$$

Now by choosing $b=c \sqrt{n} T(n))$ for any constant $c>1$, we get $\operatorname{Pr}\left(Z_{n}=\Omega(\sqrt{n} T(n))\right)=O(\exp (-\sqrt{n} T(n)))$ if $a$ is small enough.
Thus by the union bound, we have

$$
\begin{aligned}
& \operatorname{Pr}(\text { Some cell intersects } \Omega(\sqrt{n} T(n)) \text { lines }) \\
\leq & \sum_{k, j} \operatorname{Pr}\left(\text { Cell } S_{j k} \text { intersects } \Omega(n T(n)) \text { lines }\right) \\
= & O\left(\frac{n}{T^{2}(n)} \exp (-\sqrt{n} T(n))\right)
\end{aligned}
$$

The right hand side tends to zero for any value of $T(n)$.

From earlier discussion, we know that there exists a transmission schedule such that in every $L^{2}$ ( $L$ is a constant) time slots, each cell receives one time slot to transmit at rate $C_{\text {proposed }} W$ bits/second as shown in Eq. (33) with maximum transmission distance $T(n)$. So the rate at which each cell can transmit is $\log (T(n)) W / L^{2}$. From Lemma 5.3, each cell needs to transmit at rate $O(C(n) \sqrt{n} T(n))$ where $C(n)$ is the throughput capacity of the network. This can be accommodated by all cells if

$$
C(n) \sqrt{n} T(n)=\Theta\left(\log (T(n)) W / L^{2}\right)
$$

Note that in each cell, the traffic passing through that cell can be handled by any designated node in that cell. The following theorem describes the main result of this paper.

Theorem 5.4: In extended wireless ad hoc networks, the unicast throughput capacity in multipath fading environment with multi-hop communication when nodes utilize OIM is

$$
C(n)=\Theta\left(\frac{\log (T(n))}{\sqrt{n} T(n)}\right),
$$

where $T(n)=\Omega(\sqrt{\log n})$.

Next theorem presents the throughput capacity of this network in the absence of OIM.

Theorem 5.5: In extended wireless ad hoc networks, the unicast throughput capacity with multi-hop point-to-point communication is

$$
C(n)=\Theta\left(\frac{1}{\sqrt{n} T(n)}\right),
$$

where $T(n)=\Omega(\sqrt{\log n})$.

The proof procedure for this theorem is very similar to that of Theorem 5.4 except that we do not consider the OIM effect in Eq. (6). Also note that because there is no OIM, there is only a single transmission in each cell.

When $T(n)=\Omega(\sqrt{\log n}), C(n)=\Theta\left(\frac{\log \log n}{\sqrt{n \log n}}\right)$ for fading channel utilizing OIM with $\Theta(\log \log n)$ gain compared to point-to-point communications and when $T(n)=\Theta(\sqrt{n})$, $C(n)=\Omega\left(\frac{\log n}{n}\right)$ for fading channel utilizing OIM with $\Theta(\log n)$ gain compared to point-to-point communications. The capacity of these two schemes are illustrated in Fig. 4.

Next figure demonstrates theoretical and simulation results for the capacity of wireless ad hoc networks with and without OIM. The results clearly show that our theoretical results matches simulation results. The simulation has been done with $10^{4}$ nodes in the network. Note that by increasing $\sigma$, or by decreasing SINR $_{\text {tr }}$ or transmission range $T(n)$, the throughput capacity increases as predicted by our analysis. 


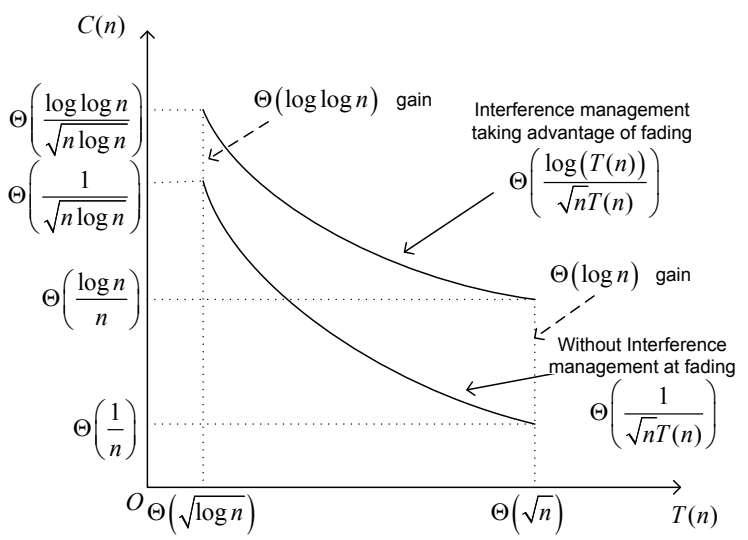

Fig. 4. The throughput capacity with and without OIM in extended wireless ad hoc network with fading channel

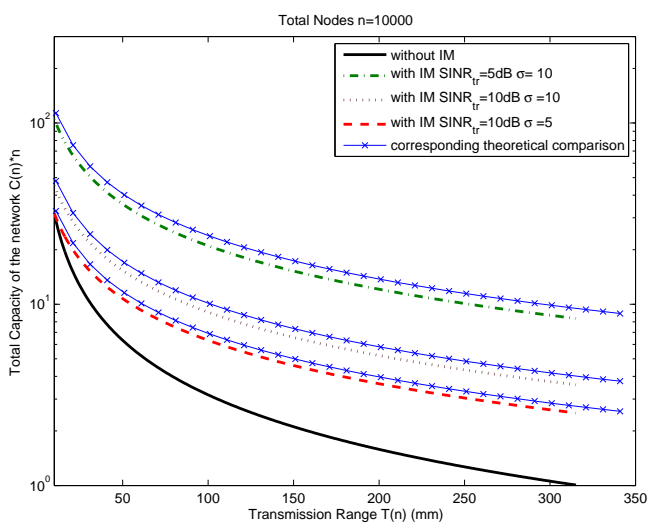

Fig. 5. The throughput capacity simulation with and without OIM as a function of $\sigma, \operatorname{SINR}_{\mathrm{tr}}$, and $T(n)$.

\section{CONCLUSION}

We introduced a new scheme called Opportunistic Interference Management (OIM) that allows multiple nodes to communicate cooperatively with each other with minimum feedback requirements. The new scheme reduces the encoding and decoding complexity of this cooperation to that of simple point-to-point communications. The new technique is an alternative to distributed MIMO systems, but with very practical feedback requirements. This scheme takes advantage of fast fluctuations of the channel due to the fading environment. We have also proved that increasing fading actually enhances the performance of the OIM scheme and increases the capacity of wireless ad hoc networks significantly compared to simple point-to-point communications.

\section{ACKNOWLEDGEMENT}

This research was partially sponsored by the U.S. Army Research Laboratory under the Network Science Collaborative Technology Alliance, Agreement Number W911NF-090053, by the Army Research Office under agreement number W911NF-05-1-0246, by the National Science Foundation under grant CCF-0729230, and by the Baskin Chair of Computer
Engineering. The views and conclusions contained in this document are those of the author(s) and should not be interpreted as representing the official policies, either expressed or implied, of the U.S. Army Research Laboratory or the U.S. Government. The U.S. Government is authorized to reproduce and distribute reprints for Government purposes notwithstanding any copyright notation hereon.

\section{REFERENCES}

[1] P. Gupta and P. Kumar, "The capacity of wireless networks," IEEE Transactions on Information Theory, vol. 46, no. 2, pp. 388-404, March 2000.

[2] A. Ozgur, O. Leveque, and D. Tse, "Hierarchical cooperation achieves optimal capacity scaling in ad hoc networks," IEEE Transactions on Information Theory, vol. 53, no. 10, pp. 2549-3572, October 2007.

[3] H. Sato, "The capacity of the gaussian interference channel under strong interference," IEEE Transactions on Information Theory, vol. 27, no. 6, pp. 786- 788, November 1981.

[4] R. Knopp and P. Humblet, "Information capacity and power control in single-cell multiuser communications," in Proc. of IEEE ICC 1995, Seattle, Washington, USA, June 18-22 1995.

[5] M. Grossglauser and D. Tse, "Mobility increases the capacity of ad hoc wireless networks," IEEE/ACM Transactions on Networking, vol. 10, no. 4, pp. 477-486, August 2002.

[6] P. Viswanath, D. Tse, and R. Laroia, "Opportunistic beamforming using dumb antennas," IEEE Transactions on Information Theory, vol. 48 , no. 6, pp. 1277-1294, June 2002.

[7] Z. Wang, M. Ji, H. Sadjadpour, and J. J. Garcia-Luna-Aceves, "Cooperation-multiuser diversity tradeoff in wireless cellular networks," in Proc. of IEEE GLOBECOM 2009, Honolulu, Hawaii, USA, November 30 - December 42009.

[8] — - "Interference management: A new paradigm for wireless cellular networks," in Proc. of IEEE MILCOM 2009, Boston, Massachusetts, USA, October 18-21 2009.

[9] V. Cadambe and S. Jafar, "Interference alignment and degrees of freedom of the K-user interference channel," IEEE Transactions on Information Theory, vol. 54, no. 8, pp. 3425-3439, August 2008.

[10] M. Sharif and B. Hassibi, "On the capacity of MIMO broadcast channels with partial side information," IEEE Transactions on Information Theory, vol. 51, no. 2, pp. 506-522, February 2005.

[11] — "A comparison of time-sharing, DPC, and beamforming for MIMO broadcast channels with many users," IEEE Transactions on Communications, vol. 55, no. 1, pp. 11-15, January 2007.

[12] L.-L. Xie and P. R. Kumar, "A network information theory for wireless communications: Scaling laws and optimal operation," IEEE Transactions on Information Theory, vol. 50, no. 5, pp. 748-767, May 2004.

[13] A. Jovicic, P. Viswanath, and S. R. Kulkarni, "Upper bounds to transport capacity of wireless networks," IEEE Transactions on Information Theory, vol. 50, no. 11, pp. 2555-2565, November 2004.

[14] S. Ahmad, A. Jovicic, and P. Viswanath, "Outer bounds to the capacity region of wireless networks," IEEE Transactions on Information Theory, vol. 52, no. 6, pp. 2770-2776, June 2006.

[15] L.-L. Xie and P. R. Kumar, "On the path-loss attenuation regime for positive cost and linear scaling of transport capacity in wireless networks," IEEE Transactions on Information Theory, vol. 52, no. 6, pp. 2313-2328, June 2006.

[16] F. Xue, L.-L. Xie, and P. R. Kumar, "The transport capacity of wireless networks over fading channels," IEEE Transactions on Information Theory, vol. 51, no. 3, pp. 834-847, March 2005.

[17] R. Motwani and P. Raghavan, Randomized Algorithms. Cambridge University Press, 1995.

[18] D. Tse and P. Viswanath, Fundamentals of Wireless Communication. Cambridge University Press, 2005.

[19] S. Kulkarni and P. Viswanath, "A deterministic approach to throughput scaling wireless networks," IEEE Transactions on Information Theory, vol. 50, no. 6, pp. 1041-1049, June 2004.

[20] F. Xue and P. Kumar, Scaling Laws for Ad-Hoc Wireless Networks: An Information Theoretic Approach. Now Publishers Inc., 2006. 